\title{
Smart Data Analytics for Machine Learning Approach in 5G Network
}

\author{
${ }^{1}$ Camilla Schaefer and ${ }^{2}$ Ana Makatsaria \\ ${ }^{1,2}$ International Bachelor in Natural Sciences, Roskilde University, Universitetsvej 1, 4000 Roskilde, Denmark \\ ${ }^{1}$ camillaschaeferden@outlook.com
}

\author{
Article Info \\ Journal of Computing and Natural Science (http://anapub.co.ke/journals/jens/jens.html) \\ Doi: https://doi.org/10.53759/181X/JCNS202101001 \\ Received 15 October 2020; Revised form 25 November 2020; Accepted 25 December 2020. \\ Available online 05 January 2021. \\ (C)2021 Published by AnaPub Publications.
}

\begin{abstract}
Psychological radio innovation can possibly ameliorate the shortage of remote assets on the grounds that unlicensed clients can utilize remote assets just on the off chance that they no affect the tasks of authorized clients. Later, psychological radio CLOUD (CogCLOUD) will be built from numerous versatile SUs associated with one another in a circulated way, which can be sent for different applications, including smart vehicle frameworks. Notwithstanding, in CogCLOUD, channel exchanging is intrinsically important at whatever point an essential client with a permit shows up on the channel. Permitting optional clients to pick an accessible channel among a large range hence empowers dependable correspondence in this unique circumstance, yet correspondence qualities, for example, bottleneck transmission capacity, RTT would change with channel switch. Because of the change, TCP needs refresh the blockage window to utilize the accessible assets. TCP CRAHN was proposed for CogCLOUD. TCP CRAHN is first assessed in quite a while the bottleneck transmission capacity then RTT changes. Considering the outcomes, TCP CoBA is proposed to additionally increase the throughput of the above use cases. TCP CoBA refreshes the cwnd dependent on accessible cradle space in transfer hub upon channel switch, just as other correspondence attributes. Through recreations, we show that contrasted and TCP CRAHN, TCP CoBA increase the throughput by up to $200 \%$.
\end{abstract}

Keywords - Pairing, Cognitive Radio Networks, Big Data, Machine Learning, Cloud Computing, Natural Science.

\section{INTRODUCTION}

A promise method to increase the survivability as well as unwavering quality of correspondence in CogCLOUD is to permit SUs to choose the correspondence fulfilling their application prerequisites from a large scope of range. In any case, since SUs consistently need to ensure no effect on PU execution, they need to take part in periodical detecting to recognize PUs, and afterward switch channels at whatever point another PU shows up. Thus, correspondence in CogCLOUD is probably going to encounter changes in qualities regarding bottleneck transmission capacity and full circle time (RTT) because of channel exchanging [1]. Considering channel change, TCP needs to adaptively refresh its window size to accomplish a productive utilization of accessible remote assets. It's accepted that the window is controlled by clog window simply because of an enormous, promoted window. This unique circumstance, TCP CRAHN is proposed as a TCP variation for a CogCLOUD. Aside from the highlights of channel exchanging and periodically detecting, TCP CRAHN's blockage control is totally same with those of existing (TCP NewReno). At the point when a channel is changed, TCP CRAHN's clog control utilizes data sent by a hand-off hub, just like the instance of XCP. On the off chance that the hand-off hub changes its channel, it would educate the TCP sender regarding when it's start and complete the process of changing just as the data transmission and connection postponement of two adjoining hubs (i.e., another channel interface). In the wake of getting this data, the TCP sender refreshes clog window fittingly. The key capacity needed in a CogCLOUD. Nonetheless, the plan expects that every hub participates in periodical detecting, the circumstance and span of periodical detecting are completely constrained by the TCP sender. Because of this reason, the TCP sender makes an impression on the hubs on the directing way. This may not be adaptable, as the hubs need to oblige numerous streams from various TCP senders [2].

Besides, it's accepted that SUs is permitted to utilize some large scope of the range, like $400 \mathrm{MHz}$ to $6 \mathrm{GHz}$, in future CogCLOUD, for a range effectiveness [3]. Be that as it may, this can cause radical changes in bottleneck bandwidth and RTT when a hub changes its channels. Accordingly, TCP CRAHN is first assessed when bottleneck data transfer capacity and RTT radically change in CogCLOUD, presenting a few issues to debase throughput execution. Then, another TCP that addresses the connected issues recognized in Fig 1. 


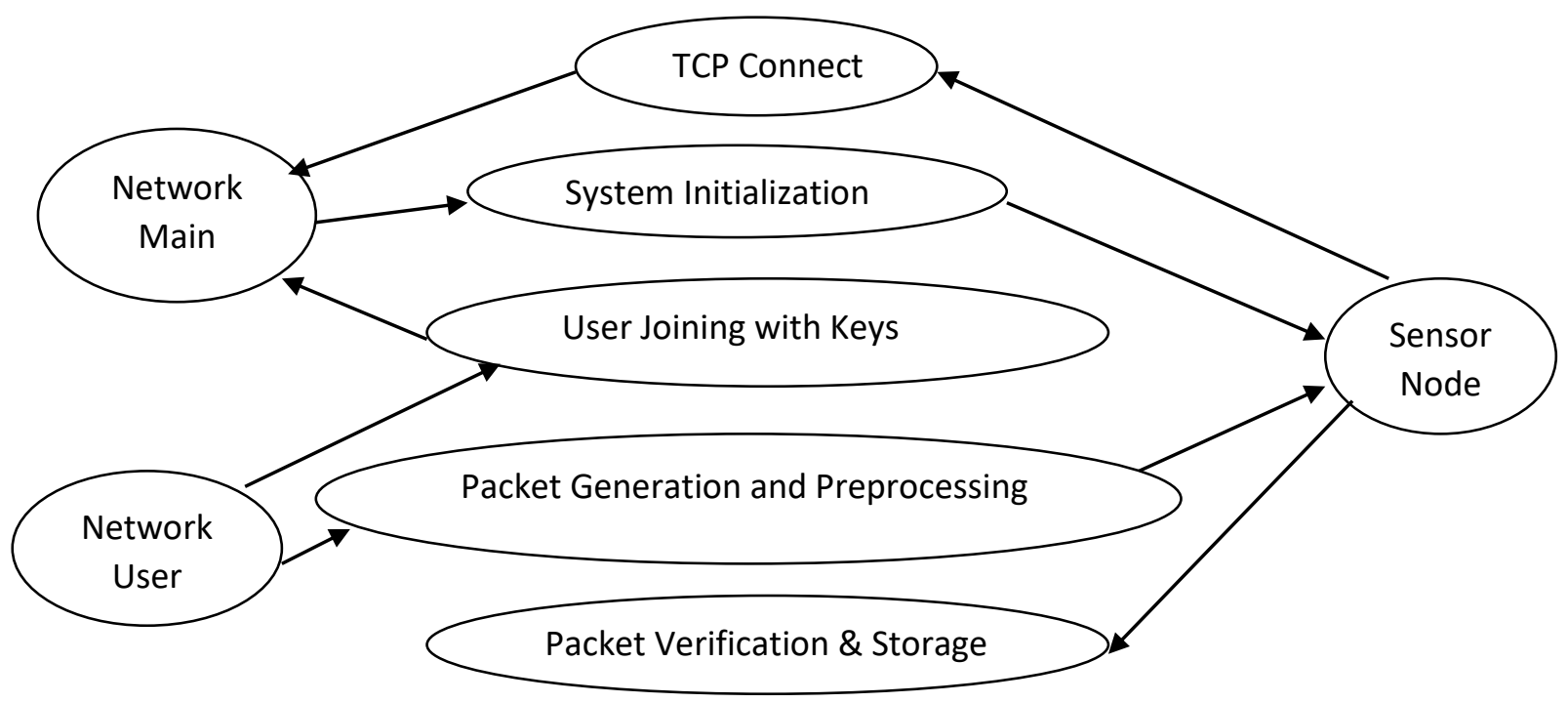

Fig 1: Architecture Diagram

\section{LITERATURE SURVEY}

\section{A. For Resource Allocation In Shared Computer Systems}

Decency is a significant exhibition basis taking all things together asset designation plans, remembering those for circulated PC frameworks. Be that as it may, it is regularly indicated just subjectively [4]. The quantity estimates in the writing are excessively explicit the specific application, experience the ill effects of some unwanted qualities. we have presented a quantity measure called Indiex of FRairness. The file is material to any asset assignment issue. It's free of measure of the asset. These decency file consistently lie somewhere in the range of 0 and 1. This boundedness helps natural comprehension of the reasonableness file. For instance, an appropriation calculation with a reasonableness of 0.10 implies that it is unjustifiable to $90 \%$ of the clients. Likewise, the segregation list can be characterized as 1 - reasonableness index.

\section{B. Intellectual radio organizations}

Range detecting is the key empowering innovation for intellectual radio organizations. The primary target of range detecting is to give more range access freedoms to intellectual radio clients without meddling with the tasks of the authorized organization. Subsequently, late examination has been centered around the obstruction shirking issue. Also, current radio recurrence (RF) front-closes cannot perform detecting and transmission simultaneously, which unavoidably diminishes their transmission openings, prompting the supposed detecting productivity issue. In this paper, to settle both the impedance evasion and the range proficiency issue, an ideal range detecting system is created [5]. More explicitly, initial a hypothetical system is created to streamline the detecting boundaries so as to boost the detecting effectiveness subject to impedance evasion imperatives. Second, to abuse various range groups, range determination and planning techniques are proposed where the best range groups for detecting are chosen to amplify the detecting limit. At last, a versatile and helpful range detecting technique is proposed where the detecting boundaries are enhanced adaptively to the quantity of collaborating users.

\section{Vehicle-to-vehicle correspondences}

Remote correspondences are getting basic to our every day life on account of the spread of different remote access innovations like cell, remote LAN, and others. Also, the wasteful utilization of the current alloted range has been brought up as a major issue because of the scant of the obliged measure of accessible radio range. At that point, intellectual radio innovation, which can adaptively distinguish spatial and fleeting changes being used over different recurrence groups is relied upon to make the remote asset sharing appropriately [6]. Then again, in impromptu interchanges, like vehicle to vehicle correspondence, the presence of a typical control channel can not accepted because of both an absence of framework and development of the hubs circulated channel coordination is perpetually required.We center around one bounce V2V corresponding the appropriated channel coordinates plot. Moreover, we build up a direct use model, which the use of each channel changes transiently and spatially. 
Dependable and high throughput information conveyance in psychological radio organizations stays an open test inferable from the failure of source to rapidly recognize and then respond to changes in range accessibility. The window-based rate variation depends on affirmations to self trigger the send rate, which are regularly deferred or lost attributable to discontinuous essential client (PU) action, bringing about an erroneous surmising of clog. The primary condition put together vehicle convention based with respect to TCP Friendly Rate Control for Cognitive Radio, which permits prompt changes in the transmission rate dependent on range related changes in organization climate [7]. TFRC-CR has the accompanying remarkable highlights: (I) use new FCC commanded range data sets with least questioning overhead, (ii)empowers change of the transmission rate by recognizing examples of genuine organization blockage, just as (iii) gives rules on when to re-start the source transmission after a delay because of PU activities.

Psychological Radio (CR) networks permit clients to entrepreneurially send in the authorized range groups, as long as the presentation of first Users of the band isn't debased. Subsequently, variety in range accessibility with time and occasional range detecting attempted by the CR clients pronouncedly affect the higher layer convention execution, for example, at vehicle layer [8]. It researches the limits of traditional TCP new Reno in a CR impromptu organization climate, and TCP CRAHN, a window-based TCP-accommodating convention. methodology fuses range mindfulness by a blend of express input from the middle of the road hubs and objective. This accomplished by adjusting traditional TCP rate control calculation run at source to intently communicate with actual layer channel data, connection layer elements of range detecting, cushion the board, prescient portability structure is created at the organization layer. An investigation of normal throughput in TCP CRAHN is given, and recreation results uncover huge upgrades by utilizing [9].

\section{Item Organizations}

Hypothesis and tests show that as the per-stream result of transfer speed and idleness builds, TCP gets wasteful and inclined to flimsiness, paying little mind to the lining plan [10]. This faltering turns out to be progressively significant as the Internet advances to consolidate extremely high-transfer speed optical connections and all the more enormous postpone satellite links. It build up a way to deal with Internet clog control that beats TCP in traditional conditions, stays proficient, reasonable, versatile, and steady as the transmission capacity defer item increments. This new eXplicit Control Protocol(XCP)sums up the Explicit Congestion Notification proposition $(\mathrm{ECN})$. This permits a more adaptable, systematically manageable convention plan and opens new roads for administration.Control hypothesis structure, Both XCP and exhibit it is steady, effective paying little mind to the connection limit, the full circle delay, and quantity of source. Broad bundle level recreations show XCP beats TCP in both ordinary and high data transfer capacity postpone conditions. Further, XCP accomplishes reasonable transfer speed allotment, high use, little standing line size, and close to zero bundle drops, with both consistent and profoundly changing traffic.

\section{PROPOSED METHODOLOGY}

TCP CRAHN is first assessed in quite a while where bottleneck transfer speed and RTT definitely change. TCP CoBA is additionally increase the throughput of the above use case. TCP CoBA refreshes the clog window dependent on accessible cushion space in transfer hub on channel switch, just as correspondence attributes. Performance debasement, brought about by exceptional change in data transmission and additionally RTT channel exchanging, can evaded viably by refreshing the clogwindow suitably shared work the hand-off hubs. The numerous TCP CoBA streams can accomplish the decent amount of the organization assets. Advantages: Our proposed work improves the throughput by up to 80 percent

\section{A. Organization Formation}

In this module, we produce intellectual radio CLOUD. This organization contains part of portable hubs and one organization regulator. All versatile hubs are associated with network regulator. The approach to increase survivability as well as the unwavering quality of correspondence in CogCLOUD is to permit SUs to choose a correspondence fulfilling prerequisites from a large scope of range.SUs consistently need to ensure no effect on PU execution,need to participate in periodically detecting to identify PUs, afterward switch channels at whatever point another PU shows up. Hence, correspondence in Cog CLOUD is probably going to encounter changes in attributes regarding bottleneck data transfer capacity and full circle time (RTT) because of channel exchanging.

\section{B. Parcel Transmission}

In this module, versatile hub finds the way for sending the parcel to the objective. First, a sender of TCP CoBA can get different data from all transfer hubs. Hand-off hubs send the data in accompanying 4 cases: three-way handshake, for-warding of information bundle, beginning of channel exchanging, and finish of channel exchanging. Finally, it send the parcel through this way. Furthermore, the TCP sender sends messages to change the circumstance and term of detecting to hubs on steering way during TCP correspondence.SU could simultaneously execute periodically detecting by abusing GPS work. CoBA is to accomplish superior by 
refreshing the clogwindow properly because of the adjustment in the bottleneck data transfer capacity (Wb) and RTT. Hence, CoBA likewise refreshes the cwnd when the RTT is changed by more than $20 \%$ because of channel exchanging, which is not quite the same as CRAHN. CoBA freezes information transmission and RTO clock during the divert exchanging as in CRAHN.

\section{Result and Experimental Setup}

To build up a framework for the future determining of the quantity of cases influenced by COVID-19 utilizing AI techniques. The dataset utilized for the examination contains data about the day by day reports of the quantity of recently contaminated cases, the quantity of recuperations, and the quantity of passings because of COVID-19 around the world. As the demise rate and affirmed cases are expanding step by step which is a disturbing circumstance for the world. The quantity of individuals who can be influenced by the COVID-19 pandemic in various nations of the world isn't notable. This examination is an endeavor to figure the quantity of individuals that can be influenced as far as new contaminated cases and passing's including the quantity of anticipated recuperations for the forthcoming 10 days. Four AI models LR, LASSO, SVM, and ES have been utilized to foresee the quantity of recently contaminated cases, the quantity of passing's, and the quantity of recoveries. The plots of affirmed cases, passing's, and recuperations on the initial four sheets followed by the plot of genuine circumstance accumulated from the real information reports of the examining time of the examination in the fifth sheet. The outcomes in the diagrams show that the ML models utilized in this examination befit the estimating task making the route towards the convenience of the investigation and future exploration of the comparative nature.

\section{CONCLUSION}

This paper zeroed in on vehicle conventions in psychological radio organization which select a channel from a range. That point inspected how the vehicle convention and the hand-off hub ought to be overhauled to utilize accessible remote asset. To start with, the TCP execution of existing TCP variations, for example, TCP CRAHN is thought about. Recreation results showed that CRAHN beats the wide range of various TCP variations considered, yet can't achieve great execution when correspondence qualities radically change because of channel exchanging. The issue emerges from unreasonably expanding window size after the divert exchanging in the above setting, which prompts numerous sequential appearances to hand-off hubs and possible support flood. Along these lines, TCP sender ought to the bottleneck hub is found and The cradle asset is accessible in the bottleneck hub, notwithstanding BDP. Then, to determine, this paper proposed TCP CoBA as another vehicle convention.SU is outfitted with a GPS capacity and subsequently can simultaneously execute periodical detecting. The sender in TCP CoBA refreshes the cwnd when either the bottleneck transmission capacity or RTT is changed by more than $20 \%$ after channel exchanging. Moreover, it likewise considers both the leftover cushion space and BDP. Through recreation tests, it was shown that, contrasted and TCP CRAHN, TCP CoBA radically increase throughput execution.

\section{References}

[1]. R. K. Jain, D.-M. W. Chiu, and W. R. Hawe, "A quantitative measure of fairness and discrimination for resource allocation and shared computer system,” Digit. Equipment Corporation, Hodson, MA, USA, Tech. Rep. DEC-TR-301 1984.

[2]. W. Y. Lee and I. F. Akyildiz, "Optimal spectrum sensing frame-work for cognitive radio networks," IEEE Trans. Wireless Com-mun., vol. 7, no. 10, pp. 3845-3857, Oct. 2008.

[3]. H. Nouraei, A. Wodoslawsky, M. Papini and J. Spelt, "Characteristics of abrasive slurry jet micro-machines: A comparison with abrasive air jet micro-machines", Journal of Materials Processing Technology, vol. 213, no. 10, pp. 1711-1724, 2013. Available: 10.1016/j.jmatprotec.2013.03.024.

[4]. S. Ramzan, "Crafting Linear Motion Problems for Problem- Based Learning Physics Classes", International Journal of Psychosocial Rehabilitation, vol. 24, no. 5, pp. 5426-5437, 2020. Available: 10.37200/ijpr/v24i5/pr2020249.

[5]. О. Вишневський, "Experimental models of monitoring abrasive wear surfaces of materials", Proceedings of National Aviation University, vol. 28, no. 2, 2006. Available: 10.18372/2306-1472.28.1342.

[6]. K. Tsukamoto, S. Matsuoka, O. Altintas, M. Tsuru, and Y. Oie, "Distributed channel coordination in cognitive wireless vehicle-tovehicle communications," in Proc. Int. Conf. Wireless Access Veh.Environ. , 2008, CD-ROM.

[7]. A. Al-Ali and K. R. Chowdhury, "TFRC-CR: An equation-based transport protocol for cognitive radio networks," Elsevier Ad Hoc Netw. J., vol. 11, no. 6, pp. 1836-1847, 2013.

[8]. K. R. Chowdhury, M. D. Felice, and I. F. Akyildiz, "TCP CRAHN: A transport control protocol for cognitive radio ad hoc networks," IEEE Trans. Mobile Comput., vol. 12, no. 4, pp. 790-803, Apr. 2013.

[9]. D. Katabi, M. Handley, and C. Rohrs, "Congestion control for high bandwidth-delay product networks," in Proc. ACM Conf. Appl., Technol., Archit. Protocols Comput. Commun., 2002, pp. 89-102.

[10]. J. Liu and S. Singh, “ATCP: TCP for CLOUD,” IEEE J. Select. Areas Commun., vol. 19, no. 7, pp. 1300-1315, Jul. 2001. 